\title{
Die Psychodynamik der Suizidalität
}

\author{
Claudius Stein
}

Online publiziert: 18. November 2019

(c) Der/die Autor(en) 2019

Zusammenfassung Wenn Menschen direkt oder indirekt mitteilen, dass sie nicht mehr leben wollen, ist dies zunächst als ein interaktionelles Geschehen zu verstehen. Daher ist das Beziehungsangebot das zentrale Element jeder Form der Behandlung, sowohl der Krisenintervention als auch der Psychotherapie suizidaler Menschen. Voraussetzung für eine konstruktive therapeutische Arbeit ist eine tragfähige Beziehung zwischen TherapeutIn und KlientIn. Damit dies gelingen kann, ist es wesentlich, die Psychodynamik und die oft subtilen Übertragungs- und Gegenübertragungsphänomene hinter der Suizidalität zu verstehen.

Suizidalität wird in tiefenpsychologischen Theorien nicht nur als ein Zeichen seelischer Dekompensation, sondern darüber hinaus als eine psychische Funktion aufgefasst. Diese wird dann eingesetzt, wenn intrapsychische oder interpersonelle Krisen nicht mehr anders handhabbar scheinen. So gesehen kann Suizidalität eine regulierende, manchmal auch stabilisierende Funktion haben. Die zentralen konflikthaften Themen des suizidalen Menschen sind der Umgang mit Aggression, die Selbstwertregulation und der Umgang mit nahen Beziehungen. Im Artikel werden die wichtigsten Theorien zur Psychodynamik der Suizidalität dargestellt.

Schlüsselwörter Suizidalität · Psychodynamik · Therapeutische Beziehung · Aggression · Narzissmus · Objektbeziehungstheorie

C. Stein $(\bowtie)$

Kriseninterventionszentrum Wien,

Lazarettgasse 14A, 1090 Wien, Österreich

claudius.stein@kriseninterventionszentrum.at

\section{The psychodynamics of suicidal tendency}

Summary When people directly or indirectly communicate that they have lost their will to live, this should initially be considered as an interactional event. Therefore, relational support is the central element of all forms of treatment, both in crisis intervention as well as the psychotherapy of suicidal people. A sustainable relationship between the therapist and the client is a prerequisite for constructive therapeutic work. In order for this to be successful, it is essential to understand the psychodynamics and the often subtle phenomena of transference and countertransference behind suicidal tendencies.

In depth psychology theories, suicidal tendency is regarded not only as a sign of emotional decompensation but also as a psychological function. This is then applied when intrapsychic or interpersonal crises seem impossible to deal with in any other way. In this respect, suicidal tendency can have a regulating and sometimes even stabilising effect. The central conflictual topics of suicidal patients are dealing with aggression, regulating self-worth and handling close relationships. The article depicts the most important theories on the psychodynamics of suicidal tendency.

Keywords Suicidal tendency · Psychodynamics · Relationship between the therapist and the client . Aggression · Narcissism · Object-relations theory

\section{Vorbemerkung}

Eine Beschäftigung mit dem Suizid aus rein psychiatrischer, psychologischer oder psychotherapeutischer Sicht wird diesem Thema nicht ausreichend gerecht. Philosophische, religiöse, gesellschaftliche und ethische Fragen weisen über eine rein klinische Betrachtungsweise hinaus. J. Améry hat in seinem bemer- 
kenswerten Buch „Hand an sich legen“ (1976) radikal und kompromisslos das Recht des Menschen auf Freitod gefordert und den Akt der Selbsttötung als letzte Freiheit des modernen Menschen bezeichnet.

Dem ist entgegenzuhalten, dass sich bei den meisten Menschen, die suizidal sind oder Suizid begangen haben, schwere psychosoziale Belastungen oder psychische Erkrankungen finden. Dies relativiert den Begriff des Freitodes, denn es stellt sich die Frage, ob ein Mensch, der - in welcher Form auch immer - leidet, in seinem Willen vollkommen frei sein kann. Von einem Bilanzsuizid spricht man, wenn Menschen subjektiv zur Einschätzung gelangen, dass ihre Lebensbedingungen so aussichtslos und unwürdig geworden sind, dass sie deshalb entscheiden, sich das Leben zu nehmen. „Der Suizid oder Suizidversuch kann dann zum letzten und schmerzlichen Ausdruck persönlicher Freiheit werden, ohne dass eine psychosoziale Fehlentwicklung oder eine körperliche Erkrankung vorgelegen hat" (Scobel 1981, S. 101). Ob es einen Bilanzsuizid in dieser gleichsam reinen Form gibt, ist schlussendlich nicht $\mathrm{zu}$ beantworten. Man findet auch bei vielen Suiziden im Rahmen von Krisen oder eindeutig diagnostizierten psychischen Erkrankungen mehr oder weniger stark ausgeprägt bilanzierende Elemente, wenn deswegen die zunehmende Einschränkung der Lebensperspektive antizipiert wird.

Die Möglichkeit über den eigenen Tod nachzudenken und den eigenen Tod aktiv herbeizuführen ist eine Realität des menschlichen Handlungsvermögens. Prinzipiell kann daher jeder Mensch Suizidgedanken entwickeln und suizidale Handlungen setzen. Suizidale Menschen sind verzweifelt, ihr Spielraum engt sich erheblich ein. Viele Betroffene glauben, dass sie auf diese Weise ihre eingeschränkten Handlungsmöglichkeiten wieder erweitern können. Wo quälende Ausweglosigkeit das Denken und Fühlen beherrscht, eröffnet sich eine neue Perspektive und ein neuer Weg. Es scheint somit oft nicht letztgültig möglich, eindeutig zu entscheiden, ob ein Suizid ausschließlich Endpunkt einer krankhaften Entwicklung ist oder doch auch ein letzter und schmerzlicher Ausdruck persönlicher Freiheit.

Solange der Mensch am Leben ist, gibt es auch andere Auswege. Suizidalität ist immer auch ein interaktionelles Geschehen. Ein tragfähiges Beziehungsangebot kann einen solchen Ausweg weisen und ist daher wesentlich für jede Form der Behandlung, sowohl der Krisenintervention als auch der Psychotherapie suizidaler Menschen. Voraussetzung für eine konstruktive psychotherapeutische Arbeit ist, dass der/die TherapeutIn das mehr oder weniger verborgene Kommunikationsangebot hinter der Suizidalität, also die Psychodynamik und die oft subtilen Übertragungsund Gegenübertragungsphänomene zu verstehen versucht (Kind 2005).

\section{Ursachen und Motivstruktur von Suizidalität}

Es gibt keine einheitliche Theorie der Suizidalität. Suizidales Verhalten ist immer das Ergebnis eines komplexen Zusammenspiels neurobiologischer, psychologischer und sozialer Faktoren und kann nur unter Berücksichtigung der Gesamtpersönlichkeit und der jeweils individuellen psychosozialen Situation und Belastung verstanden werden. TherapeutInnen müssen sich also jedes Mal aufs Neue darauf einlassen, gemeinsam mit dem/der Betroffenen die Bedeutung der suizidalen Phantasien und Handlungen zu ergründen und daraus das richtige therapeutische Handeln ableiten.

Es zeigt sich, dass es viele Menschen gibt, die auf Grund einer akuten psychosozialen Krisensituation sehr ernsthaft suizidal werden, für die dies aber die einzige suizidale Episode im Leben bleibt. Krisen werden durch äußere belastende Ereignisse wie Todesfälle, Trennungen, Krankheitsdiagnosen oder plötzlichen Arbeitsplatzverlust ausgelöst. Das Befinden Betroffener verschlechtert sich und die Situation spitzt sich rasch zu. Bewältigungsmöglichkeiten, die sonst zur Verfügung stehen, gehen verloren. Es entwickeln sich Gefühle von Verzweiflung und Hoffnungslosigkeit und verschiedene psychische und physische Symptome. Selbstwert und Identität sind in Frage gestellt und das normale psychische Funktionsniveau ist erheblich beeinträchtigt. Eine suizidale Handlung kann Betroffenen dann als letzter Ausweg erscheinen. Auch in Krisen finden sich psychodynamische Vorgänge wie sie in der Folge beschrieben werden, allerdings sind sie zeitlich begrenzt, sie bleiben also meist auf die akute Phase der Krise beschränkt. Es sind Situationen hoher Dringlichkeit, die sowohl sehr ernste Gefahren für den Betroffenen darstellen, als auch bei geglückter Bewältigung die Chance auf Reifung und Weiterentwicklung in sich bergen (Sonneck et al. 2016; Stein 2009).

Dem gegenüber geraten etwa 20 bis $30 \%$ jener Menschen, die einen Suizidversuch unternommen haben, wiederholt in suizidale Krisen. Man spricht von chronischer Suizidalität. Das Suizidrisiko von Menschen, die bereits einen oder mehrere Suizidversuche unternommen haben, ist vierzig Mal höher als in der Normalbevölkerung. Ca. 5-10\% sterben innerhalb von 10 Jahren nach dem ersten Versuch durch Suizid. Bei diesen Menschen finden sich oft sehr ernste Psychopathologien. Besonders gefährdet sind Personen mit affektiven Störungen (depressive und bipolare Störungen), mit Psychosen, Borderlineund antisozialen Persönlichkeitsstörungen, Alkohol-, Drogen- und Medikamentenmissbrauch und Essstörungen (vgl. Bronisch et al. 2002).

Die meisten Kenntnisse über die Psychodynamik suizidaler Entwicklungen resultieren aus der Auseinandersetzung mit Menschen, die Suizidgedanken haben oder Suizidversuche überlebt haben. Es gibt natürlich wenig Wissen darüber, was in Menschen 
vorging, die sich tatsächlich das Leben genommen haben. „Dem, der ihn beging, wird der Selbstmord als der letzte und beste von vielen schlechten Möglichkeiten erschienen sein und jeder Versuch der Lebenden, diesen Grenzbereich zu erkunden, kann nur eine Ahnung liefern und zum Verrücktwerden lückenhaft sein“ (Jamison 2000, S. 75). Allerdings liegt in nicht wenigen Suiziden auch eine Botschaft an die Hinterbliebenen. Man kann sagen, dass „der vollzogene Suizid den Dialog also nicht nur zerstört, sondern ihn auch wiederum eröffnet, wenn auch der Suizident selbst nicht mehr daran teilhaben kann" (Küchenhoff 2001, S. 61). Ein 23-jähriger Mann, dessen Freundin sich in Folge der von ihm initiierten Trennung in seiner Wohnung erhängt hatte, sucht therapeutische Hilfe. Er wirkt im Erstgespräch kühl und unberührt. Der Therapeut wird bei seinen Schilderungen von einem Gegenübertragungsgefühl von Entsetzen und Grauen erfasst. Gefühle, die der Klient offenbar nicht oder noch nicht ertragen kann. Allzu offensichtlich spielt bei diesem Suizid der Aspekt einer furchtbaren Rache eine Rolle.

\section{Tiefenpsychologische Theorien der Suizidalität}

In tiefenpsychologischen Theorien wird Suizidalität nicht nur als ein Zeichen seelischer Dekompensation, sondern darüber hinaus als eine psychische Funktion aufgefasst. Diese wird dann eingesetzt, wenn intrapsychische oder interpersonelle Krisen nicht mehr anders handhabbar scheinen. So gesehen kann Suizidalität eine regulierende, manchmal auch stabilisierende Funktion haben.

Die zentralen konflikthaften Themen des suizidalen Menschen sind der Umgang mit Aggression, die Selbstwertregulation und der Umgang mit nahen Beziehungen. So sieht der Psychoanalytiker Jürgen Kind (2005) die Beziehungen der Suizidanten und speziell dessen Beziehung zum Therapeuten/zur Therapeutin als eine Reinszenierung pathogener frühkindlicher Objektbeziehungen. Ein umfassendes psychodynamisches Verständnis kann also nur durch ein Zusammenführen konflikt-, selbst- und objektbeziehungspsychologischer Suizidtheorien erreicht werden.

\section{Aggressionstheoretische Aspekte der Suizidalität}

Die gemeinsame Ausgangshypothese der tiefenpsychologischen Theorie formuliert Freud in Trauer und Melancholie: „Kein Neurotiker verspürt Selbstmordabsichten, der solche nicht von einem Mordimpuls gegen andere auf sich zurückwendet“ (1917, S. 205). Er postuliert also einen Aggressionskonflikt und deutet die Suizidhandlung als Wendung der Aggression gegen die eigene Person. Diesen Konflikt versteht er als eine Reaktion auf den subjektiv unerträglichen Verlust eines realen oder phantasierten, emotional als unverzichtbar erlebten Objekts. Ein 25-jähriger Student mit einer ausgeprägten narzisstischen Problematik erlebt nach einer unglücklichen Liebe eine schwere depressive Phase mit ernsten Suizidgedanken. Er überlegt sehr konkret, von einem hohen Gebäude zu springen. Einerseits meint er ohne dieses Mädchen könne er nicht leben, denn sie ist die Liebe seines Lebens, andererseits berichtet er von einem kaum beherrschbaren Hass mit Gewaltphantasien.

\section{Narzissmustheoretische Aspekte der Suizidalität}

Noch besser verstehbar werden derartige Dynamiken durch das Konzept der narzisstischen Krise (Henseler 1984). Charakteristisch für Menschen, die eine Unsicherheit dem eigenen Selbst gegenüber haben, ist, dass sie eine unrealistische Einschätzung der eigenen Person mit einem ständigen Schwanken zwischen Größen- und Allmachtsphantasien und Minderwertigkeitsgefühlen und eine unrealistische Einschätzung der Objekte und der zwischenmenschlichen Beziehungen haben und sich daher immer wieder Tendenzen zu Idealisierung und Entwertung zeigen. PartnerInnen werden also geliebt um das Selbst zu stützen und zu befriedigen und als Teil des eigenen Selbst erlebt. Man spricht von narzisstischer Objektwahl. PartnerInnen haben somit eine äußerst wichtige Funktion für das Selbsterleben. Sie werden zu einem „Selbstobjekt“ (Kohut 1976).

Narzisstische Krisen können nicht nur durch ein vermeintliches Versagen des so geliebten Menschen ausgelöst werden, sondern z. B. auch durch berufliche Misserfolge oder durch die Einschränkungen und Bedrohungen des Älterwerdens. Immer geht es um erhebliche Kränkungen, die zu einer Schwächung des Selbstwerterlebens führen. Diese sind meist eine Wiederbelebung von Erfahrungen von Minderwertigkeit, übermächtiger Bedrohung, katastrophaler Abhängigkeit und Verlassenheit in frühen Entwicklungsphasen. Die Kränkungen lösen Gefühle massiver Enttäuschung, Ohnmacht und intensiver Wut bis hin zum Hass aus, was wiederum Schuldgefühle und Selbstbestrafungstendenzen nach sich zieht. Damit einher geht eine Entdifferenzierung von Subjekt und Objekt. Die Grenzen zwischen Subjekt und Objekt verwischen sich. Es wird unklar, woher der Hass stammt und gegen wen er sich richtet. Das Objekt wird so gerettet, gleichzeitig wird der Hass, der sich vorher auf das Objekt richtete, nunmehr gegen das Objekt im Selbst, also gegen die eigene Person gerichtet. „Der Suizid ist der psychische Mord einer Objektrepräsentanz im Subjekt durch Selbsttötung“ (Götze 2002, S. 118). Da eine überhöhte Vorstellung von der Auswirkung der eigenen Triebimpulse besteht, erscheint die Wut als etwas Bedrohliches, Unkontrollierbares. Daraus resultiert notwendigerweise eine starke Kontrolle aggressiver Gefühle.

Reichen Kompensationsmechanismen wie Realitätsverleugnung oder Selbstidealisierung mit entsprechenden Größenphantasien nicht aus um das narzisstische System $\mathrm{zu}$ restituieren, kommt es $\mathrm{zu}$ einer tiefen Regression. Der Suizid wird zu einem 
phantasierten oder agierten Rückzug auf einen harmonischen, symbiotischen Primärzustand. Subjektiv können so Wohlbehagen, Wärme und Geborgenheit zurückgewonnen werden und das Selbstgefühl wieder hergestellt werden. Die Wut bleibt diffus, wird abgespalten und gegen die eigene Person gerichtet. Die objektive Gefährlichkeit der Suizidhandlung kann subjektiv nicht mehr erkannt werden.

Suizidhandlungen sind also sehr häufig Reaktionen selbstunsicherer Menschen auf Enttäuschungen und Kränkungen. Die suizidale Phantasie hilft den Betroffenen, den subjektiv erlebten Gefühlen totaler Verlassenheit und Hilflosigkeit aktiv entgegenzutreten, um so das Selbstwertgefühl zu bewahren. In diesem Ambivalenzkonflikt geht es also sowohl um die Zerstörung des verloren geglaubten geliebten $\mathrm{Ob}$ jektes wie auch um die Rettung der Objektbeziehung. Man spricht in diesem Zusammenhang auch von einer „fusionären Suizidalität“ (Kind 2005, S. 36). In den Suizidphantasien betroffener Menschen spiegelt sich diese psychische Funktion der Suizidalität oft wieder. Eine 35-jährige Klientin kommt aufgrund einer Trennungskrise zu einem Erstgespräch. Sie sagt, dass sie an „etwas ganz Schreckliches“ dächte. Auf Nachfrage berichtet sie von sehr ernsten Suizidgedanken. Sie hat bereits Medikamente gesammelt. Wenn sie alleine zu Hause ist, denkt sie nur noch daran, sich zu töten. Sie meint, dass es ihrem 12-jährigen Sohn, mit dem sie große Konflikte hat, ohne sie auch besser gehen würde. Der Gedanke „nicht mehr zu leben" sei für sie aber gar nicht „schrecklich“, sondern eher tröstend. Sie müsste dann den inneren Schmerz nicht mehr ertragen und käme endlich zur Ruhe. Sie glaubt, dass es eine andere, bessere, harmonischere Welt gibt. Dort könnte sie auch wieder mit ihrem geliebten Mann vereint sein. Es stellt sich heraus, dass sie ihren Ehemann zwei Jahre zuvor durch einen Suizid verloren hatte. Es handelte sich um eine komplizierte Trauerreaktion mit einer ausgeprägten Idealisierung der Beziehung. Später wurde deutlich, dass diese auf Grund der Alkoholkrankheit des Mannes und erheblicher finanzieller Schwierigkeiten vor seinem Suizid eigentlich äußerst konfliktreich gewesen war. Im Rahmen der Krisenintervention konnte der Trauerprozess wiederbelebt werden und die Klientin konnte Kummer, Schuldgefühle aber auch Wut zulassen und eine innerliche Trennung vom verstorbenen Partner vollziehen, die suizidale Einengung löste sich auf.

Manche suizidale KlientInnen entwerten auf Grund ihrer narzisstischen Problematik ihre TherapeutInnen. Es besteht die Gefahr, dass diese ihre daraus resultierenden unangenehmen Gegenübertragungsgefühle wie Enttäuschung und Wut unbewusst ausagieren, indem sie den Klienten/die Klientin ihre Überlegenheit und Dominanz spüren lassen. Es entwickelt sich in der Therapie dann ein Machtkampf, der verhindert, dass es zumindest zu einer minimalen gegenseitigen Bestätigung und Akzeptanz zwischen TherapeutIn und KlientIn kommt. Diese Verbindung von Entwertung und ausbleibendem Bestätigungskreislauf gefährdet die Therapie in hohem Maße. Denn die dosierte und aufrichtige Mitteilung positiver Gegenübertragungsgefühle hat eine stabilisierende Wirkung auf suizidale narzisstische Klient_innen. Die Entwertung erschwert es TherapeutInnen aber erheblich, positive Aspekte wahrzunehmen und mitzuteilen. Wichtig ist also die Fähigkeit, „die therapeutische Beziehung auch in Momenten aggressiven Aufgeladenseins aufrecht zu erhalten" (SchneiderHeine 2017). Selbst sehr erfahrene TherapeutInnen haben oft große Schwierigkeiten, mit ablehnenden, feindseligen Reaktionen ihrer PatientInnen umzugehen. Herr K., ein 65-jähriger Klient, eröffnete das Erstgespräch mit der Aussage, er habe eine geladene Waffe zu Hause. Er vermute, dass der Therapeut ihm wohl auch nicht helfen könne so bliebe der Suizid sein einziger Ausweg. Beim Therapeuten entstanden Gefühle von Ärger, Hilflosigkeit und Ohnmacht und es tauchte unmittelbar der Wunsch auf, den Klienten rasch loszuwerden, ihn z.B. sofort stationär einzuweisen. Der Therapeut war in der Lage diese Gegenübertragungsgefühle zu reflektieren und vermied ein vorschnelles Handeln. In der Folge war ein sehr konstruktives und offenes Gespräch möglich.

\section{Objektbeziehungstheoretische Aspekte der Sui- zidalität}

Im Unterschied zum zuvor beschriebenen regressiven Rückzug, ist für manche suizidale KlientInnen die Bedrohung der Selbstgrenzen und der Autonomie das zentrale Konfliktfeld. Manche KlientInnen entwickeln Suizidphantasien, wenn sie ihre Selbstgrenzen und ihre Ich-Autonomie gefährdet sehen. Diese Form der Suizidalität dient der Abwehr von drohenden Gefühlen von Abhängigkeit, Hilflosigkeit und Kontrollverlust. Man erlebt eine derartige Dynamik nicht selten bei der Begleitung suizidaler älterer Menschen, meist Männer. Gerade im Alter muss zunehmend mit der Einschränkung von Möglichkeiten und Fähigkeiten gerechnet werden. Der tatsächliche oder vermeintliche Verlust der Selbständigkeit wird als massive Kränkung erlebt und stellt die eigene Identität in Frage. Die Entscheidung, sich zu suizidieren, erleben diese KlientInnen in ihrer Phantasie gleichsam als eine vollkommen autonome Handlung. Jürgen Kind (2005, S. 42) nennt dies die „antifusionäre“ Funktion von Suizidalität. Eine derartige Dynamik war auch bei Herrn K. der Auslöser für die Suizidalität. Einerseits erlebte er die einige Monate zurückliegende Pensionierung als eine große Kränkung. Er hatte beruflich ein sehr erfolgreiches Leben hinter sich und hatte sich erhofft, nach der Pensionierung in einer Beraterfunktion für die Firma tätig zu werden. Diese wurde aber an einen großen Investor verkauft und auf seine Expertise wurde kein Wert mehr gelegt. Er entwickelte eine schwere depressive Episode, in deren Verlauf auch körperliche Symptome auftraten, die ausgeprägte hy- 
pochondrische Ängste zur Folge hatten. Herr K. war sich sicher, schwer krank zu sein und abhängig von der Hilfe anderer zu werden. Er meinte, dass er das nicht ertragen könne. Dann sei es besser, selbst zu entscheiden und seinem Leben ein Ende zu setzen. Die Vorstellung sich zu suizidieren, erlebte dieser Klient in seiner Phantasie gleichsam als Möglichkeit, die Kontrolle über die bedrohliche Situation zurückzugewinnen.

Eine schwierige Situation in Therapien entsteht oft, wenn KlientInnen auf die Abwesenheit ihrer TherapeutInnen mit Suizidalität reagieren. Es kann sein, dass sie regelmäßig vor nahenden Urlauben mit mehr oder weniger verschlüsselten Suiziddrohungen reagieren. Sätze wie „Sie sind so lange weg, ich weiß nicht, ob ich das alleine schaffen kann." lassen in der Gegenübertragung Gefühle von Hilflosigkeit, Ohnmacht, Schuld und Ärger entstehen.

Kind nennt dies die Gegenübertragungskonstellation des „manipulierten Objektes“ (ebd., S. 127). Er spricht von manipulativer Suizidalität und vergleicht die Situation mit einer Geiselnahme. Er nennt es die Geiselnahme des Ich am Selbst. Bedroht wird zwar das Selbst, erreicht werden soll aber der Dritte, in diesem Fall der/die TherapeutIn. Unbewusst verschärfen oder lockern KlientInnen durch den Grad der Suizidalität die Kontrolle. Sie versuchen das vermeintlich sie verlassende Objekt an sich zu binden oder es zu verändern, weil sie annehmen, sie seien nicht liebenswert oder interessant genug, den so wichtigen Menschen auf andere Weise halten zu können. Die Klienten fühlen sich z.B. durch den nahenden Urlaub von unerträglichen Verlustängsten überschwemmt und entwickeln deswegen Suizidphantasien. Entscheidend für die Therapie ist, die der Objektsicherung dienenden manipulativen Aktivitäten des Patienten nicht misszuverstehen. „Wenn man in die durch den drohenden Objektverlust ausgelösten Ohnmachts- und Auslieferungsängste eintaucht, wird man am manipulativen Verhalten nichts Anrüchiges finden und nicht mehr von einem bloß demonstrativen Suizidversuch sprechen" (Kind 2011, S. 89). Gerade durch dieses Verhalten, liefern die Klienten einen Schlüssel zum Verständnis, denn genau jene Gefühle von Ohnmacht und Wut, die sie selber nur zu gut aus den Beziehungen mit ihren frühen Objekten kennen, kommen jetzt in ihren TherapeutInnen - im Sinne einer projektiven Identifikation - zur Entfaltung.

Wie es meist nicht das Ziel eines Geiselnehmers ist, seine Geisel zu töten, ist in diesem Fall nicht Ziel der Suizidalität $\mathrm{zu}$ sterben, sondern eben den Anderen $\mathrm{zu}$ halten oder zu verändern. Es geht um Objektsicherung und/oder Objektänderung (Kind 2005). In der Regel hatten diese Menschen in ihrer Kindheit niemanden, der sie bejahend und empathisch spiegelte. Sie konnten nicht die Erfahrung machen, in der Ablösung geliebt und bestätigt zu werden. Statt durch Internalisierung des äußeren empathischen Objektes eine innere Struktur der Selbstbestätigung, ein „Kernselbst“ aufzubauen, eine „Selbstrepräsen- tanz der Existenzberechtigung“ (Kind 2005), leiden sie an einer Identitätsstörung. Sie sind auf der verzweifelten Suche nach sich selbst und sind dabei auf die äußeren Objekte angewiesen und abhängig von ihnen. Immer wieder und natürlich auch in der Therapie suchen sie nach einem bejahenden guten Gegenüber. Eine 35-jährige Klientin, diagnostiziert als Borderlinepersönlichkeitsstörung, verstrickte den Therapeuten immer wieder in sehr belastende Konflikte über das Setting. So wollte sie ihn unbedingt duzen und im Kaffeehaus treffen. Ihr erschien nur die Aufgabe der Rahmenbedingungen ein ausreichender Beweis seines Interesses und seiner Wertschätzung. Mehrfach verließ sie die Therapiestunde mit einer versteckten Drohung. Sie meinte dann: „Sie wissen, heute fahre ich wieder mit dem Auto nach Hause“. Die Klientin wohnte außerhalb Wiens, anamnestisch war bekannt, dass sie bereits einmal einen fast tödlichen Autounfall hatte und mehrfach schilderte sie, wie sie einen Unfall in Kauf nehmend bei Rot über die Ampel gefahren war. Der Therapeut machte sich nach diesen Stunden große Sorgen um sie, fühlte sich ohnmächtig und hilflos, war aber auch wütend.

Wenn man in der Lage ist, solche Gegenübertragungsgefühle $\mathrm{zu}$ reflektieren, sie nicht destruktiv auszuagieren und die Beziehung zu halten, stellt dies für die KlientInnen eine wichtige korrigierende Erfahrung dar. Der/die TherapeutIn nimmt eine haltende Funktion, eine „holding function“ (Winnicott 1971) ein. Gleichzeitig sollte man dabei aber möglichst nicht erpressbar werden, d.h. gelegentlich sind bei selbstgefährdendem Verhalten auch klare Grenzen zu setzen. Wenn es also gelingt, unter Wahrung eigener Grenzen weiterhin Konstanz, empathische Wärme und Akzeptanz zu vermitteln, kann ein Verständnis für die zugrundeliegenden Verletzungen entwickelt werden und die KlientInnen entgehen irgendwann dem Zwang, immer wieder dieselbe kränkende Beziehungskonstellation herzustellen. Dann kann die Therapie zu einem konstruktiven Ende kommen. Interventionen auf dem Weg dahin sind: Klärung der auslösenden Bedingungen negativer Gefühle und der damit im Zusammenhang stehenden Objektbeziehungen, Fördern des Ertragens unangenehmer Gefühle, Verständnis für die bewussten wie unbewussten Ziele der Interaktion und Klärung wie diese Ziele auch ohne Suizidalität durch eine Veränderung der Bewältigungsstrategien erreicht werden können.

Gelingt es nicht, die haltende Funktion aufrechtzuerhalten, können KlientInnen das Gefühl entwickeln, dass alle Versuche das Objekt zu ändern oder zu halten vergeblich waren. Sie resignieren und ziehen sich zurück. Kind beschreibt diese Situation als die Gegenübertragungskonstellation des „aufgegebenen Objektes“ (ebd., S. 134). In derartigen Situationen ist es umso wichtiger, ein Stück aktiv auf die KlientInnen zuzugehen, da sie durch die weitgehende Ablösung innerer Objektbeziehungen nicht mehr selbst dazu in der Lage sind. 


\section{Schlussbemerkung}

Auf Basis des Verständnisses dieser Beziehungskonstellationen lassen sich die wesentlichen Ziele der Psychotherapie suizidaler KlientInnen formulieren. Es bedarf der projektiven Identifizierbarkeit von TherapeutInnen, d.h. man muss die Bereitschaft haben, die von KlientInnen induzierten Affekte und Phantasien zunächst aufzunehmen und sie dann weiter zu entwickeln, um sie in der Folge in weniger destruktiver Form dem therapeutischen Prozess zugänglich zu machen und den KlientInnen wieder zur Verfügung zu stellen. TherapeutInnen sollten emotional beteiligt sein und den Affekt angemessen halten können. Diese Haltung entspricht dem Prinzip des Containings (Bion 1992). Unerträgliches, nicht tolerierbares psychisches Material, oft destruktive Affekte werden von den TherapeutInnen aufgenommen und verarbeitet. Das ist nur möglich, wenn das Gespräch über den Suizid aufrechterhalten wird und es dadurch möglich wird, die Suizidphantasien in einen gemeinsamen Verstehenshorizont einzubetten (Küchenhoff 2001). Entscheidend dabei ist die gemeinsame Suche, die den KlientInnen hilft, ein neues Verständnis für sich selbst zu entwickeln. Dies ermöglicht Schritt für Schritt, die Klärung der Beziehung und transformiert den Affektsturm in eine reflexive Erfahrung (Schneider-Heine 2017). Wird der verzweifelte Versuch, ein Gegenüber zu erreichen, verstanden, dann kann es gelingen, diese Menschen im entscheidenden Moment zu halten und vor dem Sprung in die Leere $\mathrm{zu}$ bewahren und dies wird zu einer wesentlichen neuen Erfahrung für den Suizidenten, so wie es in einer berührenden Szene des Romans Riven Rock von T.C. Boyle beschrieben wird: Der Pfleger Eddie O'Kane hat eben seinen psychotischen Schützling vor dem Sprung vor einen Zug gerettet: „Er ließ Mr. Mac Cormick, der schon blutüberströmt, schon frei war, nicht unter den ratternden Rädern sterben, sondern packte ihn mit den Armen und presste ihn mit einer Kraft an sich, die keine Macht der Welt je hätte bezwingen können“ (T.C. Boyle 2000, S. 546).

Interessenkonflikt C. Stein gibt an, dass kein Interessenkonflikt besteht.

Open Access Dieser Artikel wird unter der Creative Commons Namensnennung 4.0 International Lizenz (http:// creativecommons.org/licenses/by/4.0/deed.de) veröffentlicht, welche die Nutzung, Vervielfältigung, Bearbeitung, Ver- breitung und Wiedergabe in jeglichem Medium und Format erlaubt, sofern Sie den/die ursprünglichen Autor(en) und die Quelle ordnungsgemäß nennen, einen Link zur Creative Commons Lizenz beifügen und angeben, ob Änderungen vorgenommen wurden.

\section{Literatur}

Amery, J. (1976). Hand an sich legen. Diskurs über den Freitod. Stuttgart: Klett.

Bion, W. R. (1992). Lernen durch Erfahrung. Frankfurt a. Main: Suhrkamp.

Boyle, T. C. (2000). Riven Rock. München: dtv.

Bronisch, T., Götze, P., Schmidtke, A., \& Wolfersdorf, M. (2002). Suizidalität. Ursachen - Warnsignale - therapeutische Ansätze. Stuttgart: Schattauer.

Freud, S. (1917). Trauer und Melancholie. Studienausgabe, Bd. Band III. Frankfurt a. Main: Fischer. (2000)

Götze, P. (2002). Erklärungsmodelle der psychoanalytischen Aggressions- und Narzissmustheorie. In T. Bronisch, P. Götze, A. Schmidtke \& M. Wolfersdorf (Hrsg.), Suizidalität. Ursachen - Warnsignale - therapeutische Ansätze. Stuttgart:Schattauer.

Henseler, H. (1984). Narzisstische Krisen. Zur Psychodynamik des Selbstmords. Opladen:WestdeutscherVerlag.

Jamison, K. R. (2000). Wenn es dunkel wird - Zum Verständnis des Selbstmordes. Berlin: Siedlerverlag.

Kind,J. (2005). Suizidal: Die Psychoökonomie einer Suche. Göttingen:Vandenhoeck \& Ruprecht. 1992

Kohut, H. (1976). Narzißmus. Frankfurt/Main:Suhrkamp.

Küchenhoff, J. (2001). Suizid - Suchen nach Beziehung oder Zerstörung des Dialogs. In B. Gerisch \& I. Gans (Hrsg.), Ich kehre in mich zurück und finde eine Welt-Autodestruktivität und chronische Suizdalität. Göttingen: Vandenhoeck\&Ruprecht.

Schneider-Heine, A. (2017). Krisen bei Persönlichkeitsstörungen. In Aufbruch im Umbruch - Neue Wege in der Krisenintervention. Vortrag bei der Tagung.

Scobel, W. A. (1981). Suizid-Freiheitoder Krankheit? In H.Henseler \& Ch Reimer (Hrsg.), Selbstmordgefährdung. Zur Psychodynamik und Psychotherapie. problemata, Bd. 93. Stuttgart-BadCannstatt: frommann-holzboog.

Sonneck, G., Kapusta, N., Tomandl, G., \& Voracek, M. (Hrsg.). (2016). Krisenintervention und Suizidverhütung. Wien: UTB: Facultas.

Stein, C. (2009). Spannungsfelder der Krisenintervention. Stuttgart: Kohlhammer. Neuauflage 2019

Winnicott, D.W. (1971). Vom Spiel zur Kreativität. Stuttgart: Klett-Cotta.

Hinweis des Verlags Der Verlag bleibt in Hinblick auf geografische Zuordnungen und Gebietsbezeichnungen in veröffentlichten Karten und Institutsadressen neutral. 